\title{
MONOTONICITY OF THE ERROR TERM IN GAUSS-TURÁN QUADRATURES FOR ANALYTIC FUNCTIONS
}

\author{
GRADIMIR V. MILOVANOVIĆ ${ }^{\oplus 1}$ and MIODRAG M. SPALEVIĆ 2
}

(Received March 19, 2006)

\begin{abstract}
For Gauss-Turán quadrature formulae with an even weight function on the interval $[-1,1]$ and functions analytic in regions of the complex plane which contain in their interiors a circle of radius greater than 1, the error term is investigated. In some particular cases we prove that the error decreases monotonically to zero. Also, for certain more general cases, we illustrate how to check numerically if this property holds. Some $\ell^{2}$-error estimates are considered.
\end{abstract}

2000 Mathematics subject classification: primary 41A55; secondary 65D30, 65D32.

Keywords and phrases: Gauss-Turán quadrature; error term; monotonicity; weight function; analytic functions.

\section{Introduction}

Let $w$ be an integrable weight function on the interval $(-1,1)$. It is well known that the Gauss-Turán quadrature formula with multiple nodes,

$$
\int_{-1}^{1} w(t) f(t) d t=\sum_{\nu=1}^{n} \sum_{i=0}^{2 s} A_{i, \nu} f^{(i)}\left(\tau_{\nu}\right)+R_{n, s}(f),
$$

is exact for all algebraic polynomials of degree at most $2(s+1) n-1$ and that its nodes are the zeros of the corresponding $s$-orthogonal polynomial $\pi_{n, s}(t)$ of degree $n$. For more details on Gauss-Turán quadratures and $s$-orthogonal polynomials see the book [5] and the survey paper [9]. Numerically stable procedures for calculating the Gauss-Turán quadrature formula have been proposed in $[3,10,16]$.

\footnotetext{
${ }^{1}$ Department of Mathematics, University of Niš, Faculty of Electronic Engineering, P. O. Box 73, 18000 Niš, Serbia; e-mail: grade@ni.ac.yu.

${ }^{2}$ Department of Mathematics and Informatics, University of Kragujevac, Faculty of Science,

P. O. Box 60, 34000 Kragujevac, Serbia; e-mail: spale@kg.ac.yu.

(C) Australian Mathematical Society 2007, Serial-fee code 1446-1811/07
} 
Let $\Gamma$ be a simple closed curve in the complex plane surrounding the interval $[-1,1]$ and $D$ be its interior. If the integrand $f$ is an analytic function in $D$ and continuous on $\bar{D}$, then we take as our starting point the well-known expression of the remainder term $R_{n, s}(f)$ in the form of the contour integral

$$
R_{n, s}(f)=\frac{1}{2 \pi i} \oint_{\Gamma} K_{n, s}(z) f(z) d z .
$$

The kernel is given by

where

$$
K_{n, s}(z)=\frac{\varrho_{n, s}(z)}{\left[\pi_{n, s}(z)\right]^{2 s+1}}, \quad z \notin[-1,1],
$$

$$
\varrho_{n, s}(z)=\int_{-1}^{l} \frac{\left[\pi_{n, s}(z)\right]^{2 s+1}}{z-t} w(t) d t, \quad n \in \mathbb{N},
$$

and $\pi_{n, s}(t)$ is an $s$-orthogonal polynomial with respect to the measure $w(t) d t$ on $(-1,1)$.

Let $w$ be one of the four generalized Chebyshev weight functions:
(a) $w_{1}(t)=\left(1-t^{2}\right)^{-1 / 2}$,
(b) $w_{2}(t)=\left(1-t^{2}\right)^{1 / 2+s}$,
(c) $w_{3}(t)=(1-t)^{-1 / 2}(1+t)^{1 / 2+s}$,
(d) $w_{4}(t)=(1-t)^{1 / 2+s}(1+t)^{-1 / 2}$.

It is well known that the Chebyshev polynomials of the first kind $T_{n}$ are $s$-orthogonal subject to $w_{1}(t)$ on $[-1,1]$ for each $s \geq 0$ (see [1]), and that for three other weights $w_{i}(t), i=2,3,4$, the $s$-orthogonal polynomials can be identified as Chebyshev polynomials of the second, third and fourth kinds: $U_{n}, V_{n}$ and $W_{n}$, which are defined by (see, for example, [18])

$$
U_{n}(t)=\frac{\sin (n+1) \theta}{\sin \theta}, \quad V_{n}(t)=\frac{\cos (n+1 / 2) \theta}{\cos \theta / 2} \quad \text { and } \quad W_{n}(t)=\frac{\sin (n+1 / 2) \theta}{\sin \theta / 2},
$$

respectively, where $t=\cos \theta$. However, such weights depend on $s$. The weight function in (d) can be omitted from investigation because $W_{n}(-t)=(-1)^{n} V_{n}(t)$.

Gori and Micchelli [6] have introduced for each $\dot{n}$ a class of weight functions defined on $[-1,1]$ for which the explicit Gauss-Turán quadrature formulae can be found for all $s$. In other words, these classes of weight functions have the peculiarity that the corresponding $s$-orthogonal polynomials, of the same degree, are independent of $s$. This class includes certain generalized Jacobi weight functions

$$
w_{n, \mu}(t)=\left|\frac{U_{n-1}(t)}{n}\right|^{2 \mu+1}\left(1-t^{2}\right)^{\mu},
$$


where $U_{n-1}(\cos \theta)=\sin n \theta / \sin \theta$ (a Chebyshev polynomial of the second kind) and $\mu>-1$. In this case, the Chebyshev polynomials $T_{n}(t)$ appear to be $s$-orthogonal polynomials.

Using the representation (1.2) of the remainder term $R_{n, s}(f)$, the results from $[4,8,19]$ for Gauss quadrature formulae, and taking $\Gamma$ to be a confocal ellipse or circle, some very precise error bounds of $\left|R_{n, s}(f)\right|$ are derived in [11-14]. The case with strong singularities has been considered in [15].

The monotonicity of quadrature approximations for some quadrature rules has been considered by several authors. For example, Newman [17] showed that, for a certain class of functions, the convergence of the general Newton-Cotes quadrature is monotone. Also, under the condition $f^{(2 n)} \geq 0$, Brass [2] considered the quadrature rule $Q_{n}(f)$, with $n$ nodes, and proved certain monotonicity results in the case of Gaussian and Newton-Cotes rules.

In this paper we consider the error term $R_{n, s}(f)$ in the Gauss-Turán quadrature formula, with an even weight function on the interval $[-1,1]$, for functions analytic in regions of the complex plane which contain in their interiors a circle of radius greater than 1 . For some important particular cases we prove that $R_{n, s}(f)$ decreases monotonically to zero. Also, we investigate numerically some more general cases and analyze some $\ell^{2}$-error estimates. The paper is organized as follows. In Section 2 we give some preliminary results. The monotonicity of the error term is given in Section 3 , including a numerical analysis. Finally, the $\ell^{2}$-error estimates are considered in Section 4.

\section{Preliminaries}

Let $f$ be an analytic function in $\{z:|z|<1+2 \varepsilon\}$, where $\varepsilon>0$, that is,

$$
f(z)=\sum_{k=0}^{+\infty} a_{k} z^{k}, \quad|z|<1+2 \varepsilon
$$

Let $\pi_{n, s}(t)$ be the $s$-orthogonal polynomial of the $n$th degree subject to the given weight function $w(t): \pi_{n, s}(t)=\kappa_{n} t^{n}+\kappa_{n-1} t^{n-1}+\cdots$, where we take $\kappa_{n}=\kappa_{n}^{(s)}>0$.

In the following, we suppose that $w(t)$ is even, that is, $w(-t)=w(t)$ on $[-1,1]$, and that the contour $\Gamma$ is the circle of radius $1+\varepsilon$.

The results which will be presented in this paper are a continuation of the results from [13]. Because of that we need some facts from [13], and we give them in this section again (without proofs). 
LEMMA 2.1. In the expansion

$$
\frac{1}{\pi_{n, s}(z)}=\sum_{j=0}^{+\infty} b_{n, j}^{(s)} z^{-n-j}, \quad|z| \geq 1,
$$

for $n>1$ it holds that $b_{n, 2 j+1}^{(s)}=0, b_{n, 2 j}^{(s)}>0(j=0,1,2, \ldots)$, and for $n=1$ it holds that $1 / \pi_{1, s}(z)=b_{1,0}^{(s)} z^{-1}$, where $b_{1,0}^{(s)}=1 / \kappa_{1}>0$.

Therefore, in the case with even weight function, we have

$$
\begin{aligned}
\frac{1}{\pi_{n, s}(z)} & =\sum_{j=0}^{+\infty} b_{n, 2 j}^{(s)} z^{-n-2 \jmath}, \quad|z| \geq 1, \quad \text { and } \\
\frac{1}{\left[\pi_{n, s}(z)\right]^{2 s+1}} & =z^{-n(2 s+1)}\left(\sum_{j=0}^{+\infty} b_{n, 2 j}^{(s)} z^{-2 j}\right)^{2 s+1}=\sum_{j=0}^{+\infty} \bar{b}_{n, 2 j}^{(s)} z^{-n(2 s+1)} .
\end{aligned}
$$

It is clear that $\bar{b}_{n, 2 j+1}^{(s)}=0$ and $\bar{b}_{n, 2 j}^{(s)}>0$.

If $b_{n, 2 k}^{(s)}$ are known, by using [7, Equation 0.314], it is possible to determine $\bar{b}_{n, 2}^{(s)}$ in the following way:

$$
\bar{b}_{n, 0}^{(s)}=b_{n, 0}^{2 s+1}, \quad \bar{b}_{n, 2 j}^{(s)}=\frac{1}{j \bar{b}_{n, 0}^{(s)}} \sum_{k=1}^{j}(2 k(s+1)-j) b_{n, 2 k}^{(s)} \bar{b}_{n, 2 j-2 k}^{(s)} .
$$

LEMMA 2.2. For $\varrho_{n, s}(z)$ it holds that

$$
\varrho_{n, s}(z)=\sum_{j=0}^{+\infty} c_{n, 2 j}^{(s)} z^{-n-2 j-1}, \quad|z|>1,
$$

where $c_{n, 2 \jmath}^{(s)}>0, n=0,1,2, \ldots\left(s \in \mathbb{N}_{0}\right) ; j=0,1,2, \ldots$.

THEOREM 2.3. Let $R_{n, s}(f)$ be the remainder term in the Gauss-Turán quadrature formula (1.1) and $e_{n, k}^{(s)}=\sum_{j=0}^{k} \bar{b}_{n, 2 j}^{(s)} c_{n, 2 k-2 j}^{(s)}$. Then, for $n \geq 1$,

$$
R_{n, s}(f)=\sum_{k=0}^{+\infty} a_{2 n(s+1)+2 k} e_{n, k}^{(s)}
$$

Let $\mu_{j}$ s be the moments of the weight function $w(t)$, that is, $\mu_{j}=\int_{-1}^{1} w(t) t^{j} d t$ $(j=0,1,2, \ldots)$. At the same time we introduce the quantities

$$
\bar{\mu}_{j}=\sum_{\nu=1}^{n} \sum_{i=0}^{2 s} A_{i, \nu} \frac{j !}{(j-i) !} \tau_{\nu}^{j-i}, \quad j=0,1,2, \ldots,
$$

which are obtained by applying the Gauss-Turán quadrature sum on $t^{j}$, and which can be numerically calculated by using the above mentioned procedures.

Because $e_{n, k}^{(s)}>0$, we also obtained the following consequences of Theorem 2.3. 
COROLlARY 2.4. We have

$$
\begin{aligned}
e_{n, k}^{(s)} & =\mu_{2 n(s+1)+2 k}-\bar{\mu}_{2 n(s+1)+2 k}, \quad k=0,1,2, \ldots, \quad \text { and } \\
e_{n, k}^{(s)} & =\mu_{2 n(s+1)+2 k}[1+o(1)] .
\end{aligned}
$$

Therefore, there exists $k_{0}$ such that for all $k \geq k_{0}$ it holds that $\bar{\mu}_{2 n(s+1)+2 k}>0$. Also, $\lim _{k \rightarrow+\infty} e_{n, k}^{(s)}=0$, and the sequence $\left(e_{n, k}^{(s)}\right)_{k=0,1,2, \ldots}$ is bounded.

\section{Monotonicity of the error term}

In this section we prove that the remainder $R_{n, s}(f)$ decreases monotonically to zero in some particular cases, when all $a_{2 k}$ in the expansion (2.1) are nonnegative. We use an analogous way of concluding as in Stenger [19, Lemma 4, Theorem 2] for the Gaussian quadrature formula $(s=0)$.

1. The case when $n$ is fixed. We have

$$
\begin{aligned}
& R_{n, s}(f)-R_{n, s+1}(f) \\
& \quad=\sum_{k=0}^{+\infty} a_{2 n(s+1)+2 k} e_{n, k}^{(s)}-\sum_{k=0}^{+\infty} a_{2 n(s+2)+2 k} e_{n, k}^{(s+1)} \\
& \quad=a_{2 n(s+1)} e_{n, 0}^{(s)}+\cdots+a_{2 n(s+1)+2 n-2} e_{n, n-1}^{(s)}+\sum_{k=n}^{+\infty} a_{2 n(s+1)+2 k} e_{n, k}^{(s)}-\sum_{k=0}^{+\infty} a_{2 n(s+2)+2 k} e_{n, k}^{(s+1)} \\
& =a_{2 n(s+1)} e_{n, 0}^{(s)}+\cdots+a_{2 n(s+1)+2 n-2} e_{n, n-1}^{(s)}+\sum_{k=0}^{+\infty} a_{2 n(s+1)+2 n+2 k}\left(e_{n, k+n}(s)-e_{n, k}^{(s+1)}\right) .
\end{aligned}
$$

It will hold that $R_{n, s}(f) \geq R_{n, s+1}(f), s=0,1,2, \ldots$, if it holds that

$$
e_{n, k+n}^{(s)}-e_{n, k}^{(s+1)} \geq 0, \quad k=0,1,2, \ldots
$$

Let us show that (3.1) holds if $n=1,2,3$, and $w(t)$ belongs to the class of Gori-Micchelli weight functions.

We have

$$
\begin{aligned}
e_{n, k+n}^{(s)}-e_{n, k}^{(s+1)}= & \frac{1}{2 \pi i} \oint_{\Gamma} \int_{-1}^{1} \frac{w(t) z^{2 n(s+1)+2 n+2 k}}{z-t} \frac{\left[\pi_{n, s}(t)\right]^{2 s+1}}{\left[\pi_{n, s}(z)\right]^{2 s+1}} d t d z \\
& -\frac{1}{2 \pi i} \oint_{\Gamma} \int_{-1}^{1} \frac{w(t) z^{2 n(s+2)+2 k}}{z-t} \frac{\left[\pi_{n, s+1}(t)\right]^{2 s+3}}{\left[\pi_{n, s+1}(z)\right]^{2 s+3}} d t d z .
\end{aligned}
$$


After a little reordering we obtain

$$
\begin{aligned}
e_{n, k+n}^{(s)} & -e_{n, k}^{(s+1)} \\
& =\frac{1}{2 \pi i} \oint_{\Gamma} \frac{z^{2 n(s+2)+2 k}}{\left[T_{n}(z)\right]^{2 s+3}} \int_{-1}^{1} w(t)\left[T_{n}(t)\right]^{2 s+1}\left[S_{n-1}(z, t)\left(T_{n}(z)+T_{n}(t)\right)\right] d t d z,
\end{aligned}
$$

where we put $S_{n-1}(z, t)=\left(T_{n}(z)-T_{n}(t)\right) /(z-t)$.

Finally, because of orthogonality,

$$
e_{n, k+n}^{(s)}-e_{n, k}^{(s+1)}=\frac{1}{2 \pi i} \oint_{\Gamma} \frac{z^{2 n(s+2)+2 k}}{\left[T_{n}(z)\right]^{2 s+3}} \int_{-1}^{1} w(t)\left[T_{n}(t)\right]^{2 s+2} S_{n-1}(z, t) d t d z .
$$

The value of the last expression will be equal to the coefficient of $z^{-1}$ when the expression under the integral over $\Gamma$ is expanded in the series of $z^{j}$. It is clear that all the coefficients of the series, which have been obtained by expanding $z^{2 n(s+2)+2 k} /\left[T_{n}(z)\right]^{2 s+3}$, are positive.

Since the weight function is even, the corresponding ( $s-$ ) orthogonal polynomial of degree $n$ has the form (here this is the Chebyshev polynomial of the first kind)

$$
T_{n}(t)=\alpha_{n} t^{n}-\alpha_{n-2} t^{n-2}+\alpha_{n-4} t^{n-4}-\cdots,
$$

where $\kappa_{n}=\alpha_{n}>0, \alpha_{n-2}>0, \alpha_{n-4}>0, \ldots$.

- Let $n=1$. Then $T_{1}(t)=\alpha_{1} t$ and $S_{0}(z, t)=\alpha_{1}>0$. Therefore

$$
\int_{-1}^{1} w(t)\left[T_{1}(t)\right]^{2 s+2} S_{0}(z, t) d t=\alpha_{1} \int_{-1}^{1} w(t)\left[T_{1}(t)\right]^{2 s+2} d t>0 .
$$

- Let $n=2$. Then $T_{2}(t)=\alpha_{2} t^{2}-\alpha_{0}$ and $S_{1}(z, t)=\alpha_{2}(z+t)$. Therefore

$$
\int_{-1}^{1} w(t)\left[T_{2}(t)\right]^{2 s+2} S_{1}(z, t) d t=\alpha_{2} z \int_{-1}^{1} w(t)\left[T_{2}(t)\right]^{2 s+2} d t
$$

which gives that the coefficient of $z^{-1}$ is positive.

- Let $n=3$. Then $T_{3}(t)=\alpha_{3} t^{3}-\alpha_{1} t$ and $S_{2}(z, t)=T_{3}(z) / z+\alpha_{3} t^{2}+\alpha_{3} t z$. Therefore

$$
\begin{aligned}
& \int_{-1}^{1} w(t)\left[T_{3}(t)\right]^{2 s+2} S_{2}(z, t) d t \\
& \quad=\frac{T_{3}(z)}{z} \int_{-1}^{1} w(t)\left[T_{3}(t)\right]^{2 s+2} d t+\alpha_{3} \int_{-1}^{1} w(t) t^{2}\left[T_{3}(t)\right]^{2 s+2} d t .
\end{aligned}
$$

Now, because of

$$
\int_{-1}^{1} w(t)\left[T_{3}(t)\right]^{2 s+2} d t>0 \quad \text { and } \quad \alpha_{3} \int_{-1}^{1} w(t) t^{2}\left[T_{3}(t)\right]^{2 s+2} d t>0
$$


we conclude that the coefficient of $z^{-1}$ is positive.

It is not possible, in this way, to prove positivity of the coefficient of $z^{-1}$ for $n \geq 4$.

2. The case when $s$ is fixed. Consider the generalized Chebyshev weight functions which are even, that is, $w_{1}(t)$ and $w_{2}(t)$.

We have

$$
\begin{aligned}
R_{n, s}(f)-R_{n+1, s}(f)= & \sum_{k=0}^{+\infty} a_{2 n(s+1)+2 k} e_{n, k}^{(s)}-\sum_{k=0}^{+\infty} a_{2(n+1)(s+1)+2 k} e_{n+1, k}^{(s)} \\
= & a_{2 n(s+1)} e_{n, 0}^{(s)}+\cdots+a_{2 n(s+1)+2 s} e_{n, s}^{(s)} \\
& +\sum_{k=s+1}^{+\infty} a_{2 n(s+1)+2 k} e_{n, k}^{(s)}-\sum_{k=0}^{+\infty} a_{2(n+1)(s+1)+2 k} e_{n+1, k}^{(s)} \\
= & a_{2 n(s+1)} e_{n, 0}^{(s)}+\cdots+a_{2 n(s+1)+2 s} e_{n, s}^{(s)} \\
& +\sum_{k=0}^{+\infty} a_{2 n(s+1)+2(s+1)+2 k}\left(e_{n, k+s+1}^{(s)}-e_{n+1, k}^{(s)}\right) .
\end{aligned}
$$

It will hold that $R_{n, s}(f) \geq R_{n+1, s}(f), n=0,1,2, \ldots$, if it holds that

$$
e_{n, k+s+1}^{(s)}-e_{n+1, k}^{(s)} \geq 0, \quad k=0,1,2, \ldots
$$

We have

$$
\begin{aligned}
e_{n, k+s+1}^{(s)} & -e_{n+1, k}^{(s)} \\
= & \frac{1}{2 \pi i} \oint_{\Gamma} \int_{-1}^{1} \frac{w(t) z^{2 n(s+1)+2(k+s+1)}}{z-t}\left[\left(\frac{\pi_{n, s}(t)}{\pi_{n, s}(z)}\right)^{2 s+1}-\left(\frac{\pi_{n+1, s}(t)}{\pi_{n+1, s}(z)}\right)^{2 s+1}\right] d t d z \\
= & \frac{1}{2 \pi i} \oint_{\Gamma} \int_{-1}^{1} \frac{w(t) z^{2 n(s+1)+2(k+s+1)}}{\left[\pi_{n, s}(z)\right]^{2 s+1}\left[\pi_{n+1, s}(z)\right]^{2 s+1}} \frac{\pi_{n, s}(t) \pi_{n+1, s}(z)-\pi_{n+1, s}(t) \pi_{n, s}(z)}{z-t} \\
& \times\left(\sum_{t=0}^{2 s}\left[\pi_{n, s}(t) \pi_{n+1, s}(z)\right]^{2 s-i}\left[\pi_{n+1, s}(t) \pi_{n, s}(z)\right]^{i}\right) d t d z
\end{aligned}
$$

Finally, by using the Christoffel-Darboux identity,

$$
\begin{aligned}
e_{n, k+s+1}^{(s)} & -e_{n+1, k}^{(s)} \\
= & \frac{1}{2 \pi i} \frac{\kappa_{n+1}}{\kappa_{n}} \oint_{\Gamma} \frac{z^{2 n(s+1)+2(k+s+1)}}{\left[\pi_{n}(z)\right]^{2 s+1}\left[\pi_{n+1}(z)\right]^{2 s+1}} \sum_{k=0}^{n} \sum_{i=0}^{2 s} \pi_{k}(z)\left[\pi_{n+1}(z)\right]^{2 s-i}\left[\pi_{n}(z)\right]^{i} \\
& \times \int_{-1}^{1} w(t) \pi_{k}(t)\left[\pi_{n}(t)\right]^{2 s-t}\left[\pi_{n+1}(t)\right]^{i} d t d z
\end{aligned}
$$

where $\pi_{k}$ is the corresponding ordinary orthogonal polynomial. 
Consider now the case $w(t) \equiv w_{1}(t), t \in(-1,1)$. By substituting $t=\cos \theta$ in the last integral we obtain

$$
\begin{aligned}
e_{n, k+s+1}^{(s)} & -e_{n+1, k}^{(s)} \\
= & \frac{1}{2 \pi i} \frac{\kappa_{n+1}}{\kappa_{n}} \oint_{\Gamma} \frac{z^{2 n(s+1)+2(k+s+1)}}{\left[\pi_{n}(z)\right]^{2 s+1}\left[\pi_{n+1}(z)\right]^{2 s+1}} \sum_{k=0}^{n} \sum_{i=0}^{2 s} \pi_{k}(z)\left[\pi_{n+1}(z)\right]^{2 s-i}\left[\pi_{n}(z)\right]^{i} \\
& \times \int_{0}^{\pi} \cos k \theta[\cos n \theta]^{2 s-i}[\cos (n+1) \theta]^{i} d \theta d z .
\end{aligned}
$$

We see that $\kappa_{n+1} / \kappa_{n}>0$, and that the expansions of

$$
\frac{z^{2 n(s+1)+2(k+s+1)}}{\left[\pi_{n}(z)\right]^{2 s+1-i}\left[\pi_{n+1}(z)\right]^{i+1}}, \quad i=0,1, \ldots, 2 s,
$$

in the series of $z^{j}$ are such that all their coefficients are positive.

Further, we use the following known formulae $[\cos n \theta]^{2 s-i}=\sum_{j=0}^{2 s-i} a_{j}^{(i)} \cos j n \theta$ and $[\cos (n+1) \theta]^{i}=\sum_{r=0}^{i} b_{r}^{(i)} \cos r(n+1) \theta$, and put

$$
a_{j}^{(i)}=\frac{\bar{a}_{j}^{(i)}}{\int_{0}^{\pi} \cos ^{2} j n \theta d \theta} \quad \text { and } \quad b_{r}^{(i)} \frac{\bar{b}_{r}^{(i)}}{\int_{0}^{\pi} \cos ^{2} r(n+1) \theta d \theta},
$$

where

$\bar{a}_{j}^{(i)}=\int_{0}^{\pi}[\cos n \theta]^{2 s-i} \cos j n \theta d \theta$ and $\bar{b}_{r}^{(i)}=\int_{0}^{\pi}[\cos (n+1) \theta]^{i} \cos r(n+1) \theta d \theta$.

Both $a_{j}^{(i)}$ and $b_{r}^{(i)}$ can be found by using [7, Equation 3.631-17].

Now,

$$
\begin{aligned}
\int_{0}^{\pi} \cos k \theta[\cos n \theta]^{2 s-i}[\cos (n+1) \theta]^{i} d \theta \\
=\sum_{j=0}^{2 s-1} \sum_{r=0}^{i} a_{j}^{(i)} b_{r}^{(i)} \int_{0}^{\pi} \cos k \theta \cos j n \theta \cos r(n+1) \theta d \theta \\
=\frac{1}{2} \sum_{j=0}^{2 s-i} \sum_{r=0}^{i} a_{j}^{(i)} b_{r}^{(i)} \int_{0}^{\pi}[\cos (k+j n) \theta+\cos (k-j n) \theta] \cos r(n+1) \theta d \theta \\
=\frac{1}{4} \sum_{j=0}^{2 s-i} \sum_{r=0}^{i} a_{j}^{(i)} b_{r}^{(i)} \int_{0}^{\pi}[\cos (k+j n+r+r n) \theta+\cos (k+j n-r-r n) \theta \\
\quad+\cos (k-j n+r+r n) \theta+\cos (k-j n-r-r n) \theta] d \theta .
\end{aligned}
$$

Since $w(t) \equiv w_{1}(t)$ is an even weight function, the previous integrals are different from zero in those cases when $k, i$ are both of even parity. Further, $\bar{a}_{j}^{(t)}=0$ if $i+j$ is 
odd and $\bar{b}_{r}^{(i)}=0$ if $i+r$ is odd. Therefore, we consider the cases in which $k, i, j, r$ are all of even parity. The last integrals are of the form $\int_{0}^{\pi} \cos l \theta d \theta, l \in \mathbb{Z}$, and are equal to zero, except when $l=0$.

Consider the case $s=1$. It is not difficult to conclude that the integral in (3.3) is not zero only in the cases when $(k, i, j, r)$ take the following values: $(0,0,0,0)$, $(0,2,0,0)$ and $(1,1,1,1)$. In these cases we have:

(i) If $(k, i, j, r)=(0,0,0,0)$, then

$$
\bar{a}_{0}^{(0)}=\int_{0}^{\pi} \cos ^{2} n \theta d \theta>0, \quad \bar{b}_{0}^{(0)}=\int_{0}^{\pi} d \theta>0 .
$$

(ii) If $(k, i, j, r)=(0,2,0,0)$, then

$$
\bar{a}_{0}^{(2)}=\int_{0}^{\pi} d \theta>0, \quad \bar{b}_{0}^{(0)}=\int_{0}^{\pi} \cos ^{2}(n+1) \theta d \theta>0 .
$$

(iii) If $(k, i, j, r)=(1,1,1,1)$, then

$$
\bar{a}_{1}^{(1)}=\int_{0}^{\pi} \cos ^{2} n \theta d \theta>0, \quad \bar{b}_{1}^{(1)}=\int_{0}^{\pi} \cos ^{2}(n+1) \theta d \theta>0 .
$$

Therefore, we conclude that the requested coefficient of $z^{-1}$ will be positive.

We derived the same conclusion in the case when $w(t) \equiv w_{2}(t)$.

Concluding in an analogous way we cannot see what happens if $s=2$. All that we can conclude is that $R_{1,2}(f) \geq R_{2,2}(f)$.

3.1. Numerical analysis The formulae (3.1) and (3.2) give us a way to check numerically if monotonicity holds, for the error in the Gauss-Turán quadrature formulae in the cases under consideration in this paper.

Let us consider the Legendre weight function $w(t)=w_{L}(t) \equiv 1$ on $[-1,1]$. It is possible to display the graphs of $e_{n, k+n}^{(s)}$ and $e_{n, k}^{(s+1)}$ for $0 \leq k \leq M$, for $M$ sufficiently large, by using programme packages such as MATLAB or MATHEMATICA, and so checking the conditions (3.1). The same can be said for the conditions (3.2).

Let $n$ be fixed, for instance, $n=4$. In Figure 1 we see the graphs of $e_{4, k+4}^{(s)}$ (connected by dashed lines) and $e_{4, k}^{(s+1)}$ (connected by solid lines), for $s=1$ (left) and $s=2$ (right), where $k=0,1,2, \ldots, 70$.

Finally let $s$ be fixed, for instance, $s=1$. In Figure 2 we see the graphs of $e_{n, k+2}^{(1)}$ (connected by dashed lines) and $e_{n+1, k}^{(1)}$ (connected by solid lines), for $n=3$ (left) and $n=4$ (right), where $k=0,1,2, \ldots, 60$. 

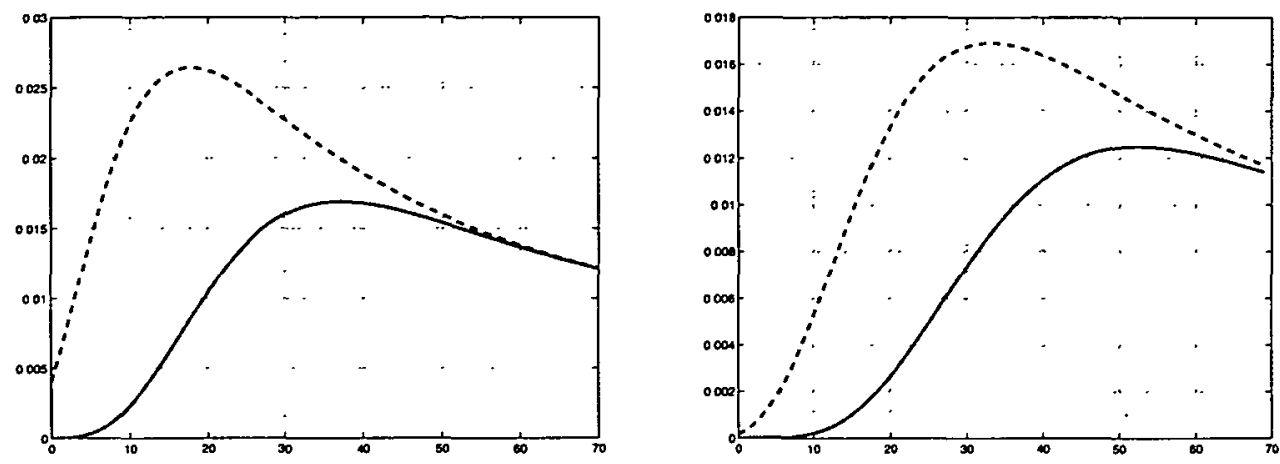

FIGURE 1. The graphs show: $R_{4,1} \geq R_{4,2}$ (left) and $R_{4,2} \geq R_{4,3}$ (right).
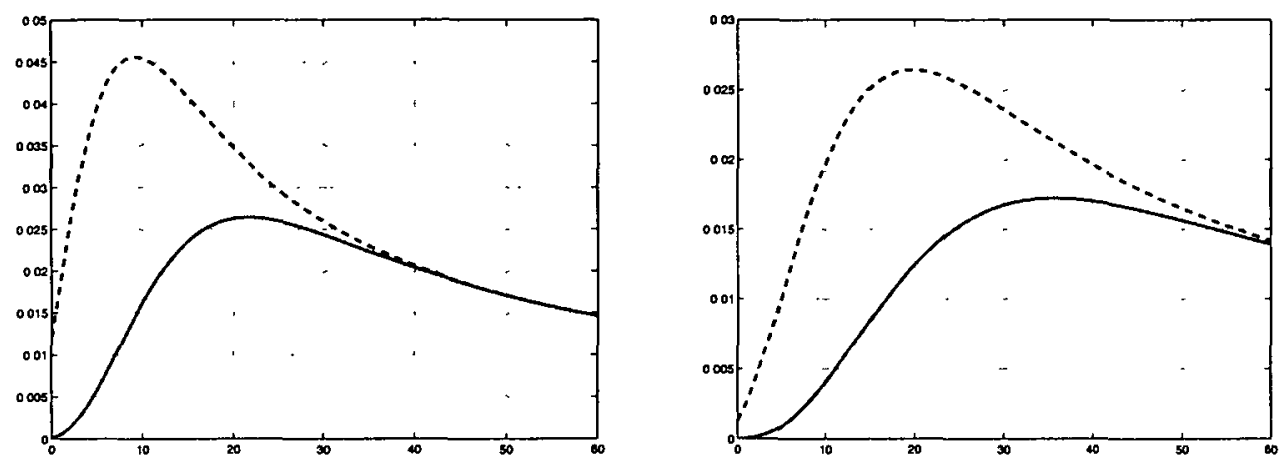

FIGURE 2. The graphs show: $R_{3,1} \geq R_{4,1}$ (left) and $R_{4,1} \geq R_{5,1}$ (right).

\section{Some $\ell^{2}$-error estimates}

For the remainder term in the Gauss-Turán quadrature formula (1.1) we can derive the following estimate:

$$
\left|R_{n, s}(f)\right| \leq\left\|R_{n, s}\right\|\|f\| .
$$

If a given sequence $\left(e_{n, k}^{(s)}\right)_{k=0,1 \ldots}\left(n, s\right.$ fixed) belongs to the space $\ell^{p}(p \geq 1)$, we apply Hölder's inequality to (2.2) to obtain

$$
\left|R_{n, s}(f)\right| \leq\left(\sum_{k=0}^{+\infty}\left(e_{n, k}^{(s)}\right)^{p}\right)^{1 / p}\left(\sum_{k=0}^{+\infty}\left|a_{2 n(s+1)+2 k}\right|^{q}\right)^{1 / q}
$$

where $1 / p+1 / q=1$.

The quantities $\sigma_{n, p}^{(s)}=\left(\sum_{k=0}^{+\infty}\left(e_{n, k}^{(s)}\right)^{p}\right)^{1 / p}$ are independent of $f$ and can be computed. The case when $p \rightarrow+\infty$ was considered in [13]. 
TABLE 1. Error constants $W_{n, s}^{1 / 2}\left(w_{i}\right)(i=L, 2)$ for some $n, s$ and for the weight functions $w_{L}(t)=1$, $w_{2}(t)=\sqrt{1-t^{2}}, t \in[-1,1]$.

\begin{tabular}{|r|l|l||l|l|}
\hline$n$ & $W_{n, 1}^{1 / 2}\left(w_{L}\right)$ & $W_{n, 2}^{1 / 2}\left(w_{L}\right)$ & $W_{n, 1}^{1 / 2}\left(w_{2}\right)$ & $W_{n, 2}^{1 / 2}\left(w_{2}\right)$ \\
\hline \hline 5 & $1.58(-1)$ & $1.24(-1)$ & $1.86(-2)$ & $1.11(-2)$ \\
\hline 6 & $1.33(-1)$ & $1.04(-1)$ & $1.33(-2)$ & $7.88(-3)$ \\
\hline 7 & $1.14(-1)$ & $8.93(-2)$ & $9.99(-3)$ & $5.88(-3)$ \\
\hline 8 & $1.01(-1)$ & $7.84(-2)$ & $7.77(-3)$ & $4.55(-3)$ \\
\hline 9 & $8.97(-2)$ & $6.98(-2)$ & $6.22(-3)$ & $3.63(-3)$ \\
\hline 10 & $8.09(-2)$ & $6.30(-2)$ & $5.09(-3)$ & $2.96(-3)$ \\
\hline
\end{tabular}

Here we consider the case $p=q=2$. The estimate (4.2) of type (4.1) gives

$$
\left\|R_{n, s}\right\|=\left(\sum_{k=0}^{+\infty}\left(e_{n, k}^{(s)}\right)^{2}\right)^{1 / 2}=W_{n, s}^{1 / 2}
$$

On the basis of the results from Section 2, there exists a $k_{0}$ such that

$$
0<\bar{\mu}_{2 n(s+1)+2 k} \leq \mu_{2 n(s+1)+2 k}, \quad k=k_{0}, k_{0}+1, \ldots
$$

that is,

$$
\bar{\mu}_{2 n(s+1)+2 k}^{2} \leq \mu_{2 n(s+1)+2 k}^{2}, \quad k \geq k_{0} .
$$

Therefore, the series $\sum_{k=0}^{+\infty} \bar{\mu}_{2 n(s+1)+2 k}^{2}$ converges if the series $\sum_{k=0}^{+\infty} \mu_{2 k}^{2}$ converges. But the last series converges if and only if the integral

converges, since

$$
I=\int_{-1}^{1} \int_{-1}^{1} \frac{w(t) w(y)}{1-t y} d t d y
$$

$$
\sum_{k=0}^{+\infty} \mu_{2 k}^{2}=\sum_{k=0}^{+\infty} \int_{-1}^{1} w(t) t^{2 k} d t \int_{-1}^{1} w(y) y^{2 k} d y=I .
$$

Therefore, if the integral $I$ converges, then the quantities $W_{n, s}^{1 / 2}=W_{n, s}^{1 / 2}(w)$ exist and can be calculated. The error constants $W_{n, s}^{1 / 2}\left(w_{i}\right)(i=L, 2)$ for some values of $n, s$ and for weight functions $w_{L}(t)=1, w_{2}(t)=\sqrt{1-t^{2}}, t \in[-1,1]$, are displayed in Table 1. Numbers in parentheses indicate decimal exponents. These do not exist for the weight function $w_{1}(t)=1 / \sqrt{1-t^{2}}$ (see, for example [19]). A calculation of $W_{n, 0}^{1 / 2}$ in (4.3) for the Gaussian rule was given by Wilf [20] and Stenger [19, table on page 157].

We finish, by deriving another expression for $W_{n, s}$. Formula (4.2), for $p=q=2$, 
has been derived as follows:

$$
\begin{aligned}
\left|R_{n, s}(f)\right| & =\left|\int_{-1}^{1} w(t) f(t) d t-\sum_{\nu=1}^{n} \sum_{t=0}^{2 s} A_{i, v} f^{(i)}\left(\tau_{v}\right)\right| \\
& =\left|\int_{-1}^{1} w(t)\left(\sum_{j=0}^{+\infty} a_{j} t^{j}\right) d t-\sum_{v=1}^{n} \sum_{i=0}^{2 s} A_{i, \nu}\left(\sum_{j=0}^{+\infty} a_{j} t^{j}\right)_{\tau_{\nu}}^{(t)}\right| \\
& =\left|\sum_{j=0}^{+\infty} a_{j}\left[\mu,-\sum_{v=1}^{n} \sum_{i=0}^{2 s} A_{i, v}\left(t^{j}\right)_{\tau_{v}}^{(i)}\right]\right| \\
& =\left|\sum_{j=2 n(s+1)}^{+\infty} a_{j}\left(\mu_{j}-\bar{\mu}_{j}\right)\right| \\
& \leq\left(\sum_{j=2 n(s+1)}^{+\infty}\left|a_{j}\right|^{2}\right)^{1 / 2}\left(\sum_{j=2 n(s+1)}^{+\infty}\left(\mu_{j}-\bar{\mu}_{j}\right)^{2}\right)^{1 / 2} .
\end{aligned}
$$

Equality can be obtained in (4.4) by putting

$$
a_{j}= \begin{cases}0, & j=0,1, \ldots, 2 n(s+1)-1, \\ \mu_{j}-\bar{\mu}_{j}, & j=2 n(s+1), 2 n(s+1)+1, \ldots,\end{cases}
$$

that is, for the function

$$
f(t)=\sum_{j=2 n(s+1)}^{+\infty}\left(\mu_{j}-\bar{\mu}_{j}\right) t^{j} .
$$

We have that (subject to (4.5), $a_{j}=0$ always when $j$ is odd, since $\mu_{j}=\bar{\mu}_{j}=0$ ) for the function $f$ in (4.6) it holds that

$$
a_{j}=a_{2 n(s+1)+2 k}=e_{n, k}^{(s)}>0, \quad j=2 n(s+1), 2 n(s+1)+2, \ldots
$$

On the basis of these facts and (4.5) we conclude that

$$
a_{j} \geq 0, \quad j=0,1, \ldots
$$

By using (4.4), where $a_{j}$ are given by (4.5), we have

$$
\begin{aligned}
W_{n, s}= & \mid \int_{-1}^{1} w(t) \sum_{j=2 n(s+1)}^{+\infty}\left[\mu_{j}-\sum_{\nu=1}^{n} \sum_{i=0}^{2 s} A_{i, \nu} \frac{j !}{(j-i) !} \tau_{\nu}^{j-i}\right] t^{j} d t \\
& -\sum_{\nu=1}^{n} \sum_{i=0}^{2 s} A_{i, \nu}\left(\sum_{j=2 n(s+1)}^{+\infty}\left[\mu_{j}-\sum_{v_{1}=1}^{n} \sum_{i_{1}=0}^{2 s} A_{i_{1}, \nu_{1}} \frac{j !}{\left(j-i_{1}\right) !} \tau_{v_{1}}^{j-i_{1}}\right] t^{j}\right)_{\tau_{\nu}}^{(i)} \mid .
\end{aligned}
$$


In fact, on the basis of (4.4) and (4.7), $W_{n, s}$ is equal to the last expression inside the absolute value, that is,

$$
\begin{aligned}
W_{n, s}= & \int_{-1}^{1} w(t) \sum_{j=2 n(s+1)}^{+\infty}\left[\mu,-\sum_{\nu=1}^{n} \sum_{i=0}^{2 s} A_{i, \nu} \frac{j !}{(j-i) !} \tau_{\nu}^{j-i}\right] t^{j} d t \\
& -\sum_{\nu=1}^{n} \sum_{i=0}^{2 s} A_{i, \nu}\left(\sum_{j=2 n(s+1)}^{+\infty}\left[\mu_{j}-\sum_{\nu_{1}=1}^{n} \sum_{i_{1}=0}^{2 s} A_{i_{1}, \nu_{1}} \frac{j !}{\left(j-i_{1}\right) !} \tau_{\nu_{1}}^{j-i_{1}}\right] t^{j}\right)_{\tau_{v}}^{(i)}
\end{aligned}
$$

Let us put the last formula in order. First, we have

$$
\begin{aligned}
\int_{-1}^{1} w(t) & \sum_{j=2 n(s+1)}^{+\infty} \mu, t^{j} d t \\
& =\int_{-1}^{1} w(t)\left[\int_{-1}^{1} w(y)\left(t^{2 n(s+1)} y^{2 n(s+1)}+t^{2 n(s+1)+1} y^{2 n(s+1)+1}+\cdots\right) d y\right] d t \\
& =\int_{-1}^{1} w(t) t^{2 n(s+1)} d t \int_{-1}^{1} w(y) y^{2 n(s+1)}(1+t y+\cdots) d y \\
& =\int_{-1}^{1} \int_{-1}^{1} \frac{w(t) w(y)(t y)^{2 n(s+1)}}{1-t y} d t d y .
\end{aligned}
$$

Second, we have

$$
\begin{gathered}
\int_{-1}^{1} w(t) \sum_{j=2 n(s+1)}^{+\infty}\left[-\sum_{\nu=1}^{n} \sum_{i=0}^{2 s} A_{i, \nu} \frac{j !}{(j-i) !} \tau_{\nu}^{j-i}\right] t^{j} d t \\
-\sum_{\nu=1}^{n} \sum_{i=0}^{2 s} A_{i, \nu} \sum_{j=2 n(s+1)}^{+\infty} \mu_{j} \frac{j !}{(j-i) !} \tau_{\nu}^{j-i} \\
=-2 \sum_{\nu=1}^{n} \sum_{i=0}^{2 s} A_{i, \nu} \sum_{j=2 n(s+1)}^{+\infty} \mu_{j} \frac{j !}{(j-i) !} \tau_{\nu}^{j-i} .
\end{gathered}
$$

We now determine the last sum in this expression.

Since $(1-\xi)^{-(l+1)}=\sum_{j=l}^{+\infty}\left(\begin{array}{l}j \\ l\end{array}\right) \xi^{j-l},|\xi|<1$, we have

$$
\begin{aligned}
& \sum_{j=2 n(s+1)}^{+\infty} \frac{j !}{(j-i) !} \tau_{\nu}^{j-i} \mu, \\
& \quad=i ! \sum_{m=2 n(s+1)}^{+\infty} \frac{j !}{i !(j-i) !} \tau_{\nu}^{j-i} \int_{-1}^{1} w(y) y^{m} d y
\end{aligned}
$$




$$
\begin{aligned}
& =i ! \int_{-1}^{1} w(y) y^{i}\left[\sum_{j=i}^{+\infty}\left(\begin{array}{l}
j \\
i
\end{array}\right)\left(\tau_{v} y\right)^{j-i}-\sum_{j=i}^{2 n(s+1)-1}\left(\begin{array}{c}
m \\
i
\end{array}\right)\left(\tau_{\nu} y\right)^{m-i}\right] d y \\
& =i !\left[\int_{-1}^{1} \frac{w(y) y^{i} d y}{\left(1-\tau_{\nu} y\right)^{i+1}}-\sum_{j=i}^{2 n(s+1)-1}\left(\begin{array}{l}
j \\
i
\end{array}\right) \tau_{\nu}^{j-i} \mu_{j}\right] .
\end{aligned}
$$

Therefore

$$
\begin{aligned}
& -2 \sum_{\nu=1}^{n} \sum_{i=0}^{2 s} A_{i, \nu} \sum_{j=2 n(s+1)}^{+\infty} \mu_{j} \frac{j !}{(j-i) !} \tau_{v}^{j-i} \\
& \quad=-2 \sum_{\nu=1}^{n} \sum_{i=0}^{2 s} i ! A_{i, \nu}\left[\int_{-1}^{1} \frac{w(y) y^{i} d y}{\left(1-\tau_{v} y\right)^{i+1}}-\sum_{j=i}^{2 n(s+1)-1}\left(\begin{array}{l}
j \\
i
\end{array}\right) \tau_{v}^{j-i} \mu_{j}\right] .
\end{aligned}
$$

Finally, we have

$$
\begin{aligned}
& \sum_{\nu=1}^{n} \sum_{i=0}^{2 s} \sum_{v_{1}=1}^{n} \sum_{i_{1}=0}^{2 s} A_{i, \nu} A_{i_{1}, \nu_{1}}\left(\sum_{j=2 n(s+1)}^{+\infty} \frac{(j !)^{2}}{(j-i) !\left(j-i_{1}\right) !} \tau_{\nu}^{j-i} \tau_{v_{1}}^{j-i_{1}}\right) \\
& =\sum_{j=2 n(s+1)}^{+\infty}\left[\sum_{\nu=1}^{n} \sum_{i=0}^{2 s} A_{i, \nu} \frac{j !}{(j-i) !} \tau_{\nu}^{j-i}\right]^{2} \\
& =\sum_{j=2 n(s+1)}^{+\infty} \bar{\mu}_{j}^{2} .
\end{aligned}
$$

On the basis of the previous analysis, the quantity $W_{n, s}$ can be given in the form

$$
W_{n, s}=\widetilde{W}_{n, s}+\sum_{J=2 n(s+1)}^{+\infty} \bar{\mu}_{j}^{2},
$$

where

$$
\begin{aligned}
\widetilde{W}_{n, s}= & \int_{-1}^{1} \int_{-1}^{1} \frac{w(t) w(y)(t y)^{2 n(s+1)}}{1-t y} d t d y \\
& -2 \sum_{\nu=1}^{n} \sum_{i=0}^{2 s} i ! A_{i, v}\left[\int_{-1}^{1} \frac{w(y) y^{i} d y}{\left(1-\tau_{\nu} y\right)^{i+1}}-\sum_{j=i}^{2 n(s+1)-1}\left(\begin{array}{l}
j \\
i
\end{array}\right) \tau_{v}^{j-1} \mu_{j}\right] .
\end{aligned}
$$

\section{Acknowledgements}

The work was supported in part by the Serbian Ministry of Science and Environmental Protection. 
[15] Monotonicity of the error term in Gauss-Turán quadratures for analytic functions

\section{References}

[1] S. Bernstein, "Sur les polynomes orthogonaux relatifs à un segment fini", J. Math. Pures Appl. 9 (1930) 127-177.

[2] H. Brass, "Monotonie bei den Quadraturverfahren von Gauss and Newton-Cotes", Numer. Math. 30 (1978) 349-354.

[3] W. Gautschi and G. V. Milovanović, " $S$-orthogonality and construction of Gauss-Turán type quadrature formulae", J. Comput. Appl. Math. 86 (1997) 205-218.

[4] W. Gautschi and R. S. Varga, "Error bounds for Gaussian quadrature of analytic functions", SIAM J. Numer. Anal. 20 (1983) 1170-1186.

[5] A. Ghizzetti and A. Ossicini, Quadrature Formulae (Akademie Verlag, Berlin, 1970).

[6] L. Gori and C. A. Micchelli, "On weight functions which admit explicit Gauss-Turán quadrature formulas", Math. Comp. 69 (1996) 269-282.

[7] I. S. Gradshteyn and I. M. Ryzhik, Table of Integrals, Series, and Products (Academic Press, New York, 1980).

[8] D. B. Hunter, "Some error expansions for Gaussian quadrature", BIT 35 (1995) 64-82.

[9] G. V. Milovanović, "Quadratures with multiple nodes, power orthogonality, and momentpreserving spline approximation”, J. Comput. Appl. Math. 127 (2001) 267-286.

[10] G. V. Milovanović and M. M. Spalević, "Quadrature formulae connected to $\sigma$-orthogonal polynomial", J. Comput. Appl. Math. 140 (2002) 619-637.

[11] G. V. Milovanović and M. M. Spalević, "Error bounds for Gauss-Turán quadrature formulae of analytic functions", Math. Comp. 72 (2003) 855-187.

[12] G. V. Milovanović and M. M. Spalević, "Error analysis in some Gauss-Turán-Radau and GaussTurán-Lobatto quadratures for analytic functions", J. Comput. Appl. Math. 164-165 (2004) 569586.

[13] G. V. Milovanović and M. M. Spalevvić, "Bounds of the error of Gauss-Turán-type quadratures", J. Comput. Appl. Math. 178 (2005) 333-346.

[14] G. V. Milovanović and M. M. Spalević, "An error expansion for Gauss-Turán quadratures and $L^{1}$-estimates of the remainder term", $B I T 45$ (2005) 117-136.

[15] G. V. Milovanović and M. M. Spalević, "Quadrature rules with multiple nodes for evaluating integrals with strong singularities", J. Comput. Appl. Math. 189 (2006) 689-702.

[16] G. V. Milovanović, M. M. Spalević and A. S. Cvetković, "Calculation of Gaussian type quadratures with multiple nodes", Math. Comput. Modelling 39 (2004) 325-347.

[17] D. J. Newman, "Monotonicity of quadrature approximations", Proc. Amer. Math. Soc. 42 (1974) 251-257.

[18] A. Ossicini and F. Rosati, "Funzioni caratteristiche nelle formule di quadratura gaussiane con nodi multipli", Boll. Unione Mat. Ital. 11 (1975) 224-237.

[19] F. Stenger, "Bounds on the error of Gauss-type quadratures", Numer. Math. 8 (1966) 150-160.

[20] H. Wilf, "Exactness conditions in numerical quadrature", Numer. Math. 6 (1964) 315-319. 